\title{
Une pompe pour la sécrétion proximale de protons
}

Une ATPase membranaire spécifique contribue à la sécrétion luminale de protons et à la réabsorption des bicarbonates dans le tubule proximal. Lorsque la polarité d'insertion de cette protéine est inversée, celle-ci acidifie les vésicules de membranes de la bordure en brosse en présence d'ATP. Les caractéristiques pharmacologiques de cette pompe la classent parmi les $\mathrm{H}^{+}$-ATPases de type vacuolaire. La mesure de la respiration cellulaire inhibée par la bafilomycine $A_{1}$ démontre que cette activité requiert près de la moitié de l'ATP synthétisé dans les tubules proximaux des carnivores comme le chien, mais beaucoup moins chez les herbivores comme le lapin et le hamster. Ces travaux mettent en lumière la contribution variable de la $\mathrm{H}^{+}$-ATPase et de l'échangeur $\mathrm{Na}^{+}: \mathrm{H}^{+}$à la sécrétion proximale de protons selon les espèces.

\section{Josette Noël Patrick Vinay}

\section{ADRESSE}

Josette Noël : Chercheur. Groupe de recherche en transport membranaire. Département de médecine et de physiologie. Patrick Vinay : Professeur. Faculté de médecine. Université de Montréal et hôpital Notre-Dame, Québec, Canada. a concentration de bicarbonate dans l'eau plasmatique est maintenue à $25 \mathrm{mM}$ chez l'homme et le capital total de bicarbonate est approximativement de 875 mmoles dans l'eau de l'organisme (espace de distribution d'environ $50 \%$ du poids corporel). Comme 180 litres de liquide plasmatique sont filtrés par jour au niveau du rein chez cette espèce, une quantité de 4500 mmoles (180 L*25 mM) de bicarbonate est également filtrée et doit être entièrement réabsorbée pour maintenir constant le capital en bicarbonate. Cela se fera en grande partie dans le tubule proximal (revues par [1] et [2]). Contrairement à d'autres solutes réabsorbés tels les acides aminés, les phosphates, le glucose, qui bénéficient de mécanismes de transport direct par cotransport avec du $\mathrm{Na}^{+}$, la réabsorption des bicarbonates est la conséquence d'une sécrétion locale de protons. Le résultat net en est la translocation d'un ion bicarbonate du fluide tubulaire vers le milieu cellulaire proximal (figure 1). L'ion bicarbonate rejoint ensuite le milieu extracellulaire en franchissant la membrane basolatérale via des systèmes de transport facilité. Comme la sortie de bicarbonate via la membrane basolatérale n'est jamais limitante [1], la régulation de la réabsorption proximale de bicarbonate sera principalement assurée par les mécanismes initiaux de sécrétion de protons au niveau de la membrane de la bordure en brosse (MBB).

Trois mécanismes présents dans la MBB sont capables d'opérer une translocation de protons du milieu intracellulaire vers la lumière tubulaire (figure 1). Le premier, et le plus étudié de ces mécanismes, est un échangeur sodium-proton $\left(\mathrm{Na}^{+} / \mathrm{H}^{+}\right)$ ([3] ; revues par [2] et [4]). Cet échange électroneutre n'est pas 


\section{RÉFÉRENCES}

1. Alpern RJ. Cell mechanisms of proximal tubule acidification. Physiol Rev $1990 ; 70$ : 79-114.

2. Burckhardt G, Warnock DG. Mechanism of $\mathrm{H}^{+}$secretion in the proximal convoluted tubule. Sem in Nephrology $1990 ; 10$ : 93-103.

3. Murer H, Hopfer U, Kinne R. Sodium/proton antiport in brush-border membrane vesicles isolated from rat smal intestine and kidney. Biochem J 1976 ; 154 : 597-604

4. Aronson PS. Mechanisms of active $\mathrm{H}^{+}$ secretion in the proximal tubule. $A m J$ Physiol 1983 ; 245 : F647-F59.

5. Nakhoul NL, Lopes AG, Chaillet JR, Boron WF. Intracellular $\mathrm{pH}$ regulation in the $\mathrm{S}_{3}$ segment of the rabbit proximal tubule in $\mathrm{HCO}_{3}^{-}-$free solutions. $J$ Gen Physiol 1988 ; 92 : 395-412.

6. Kinne-Saffran E, Kinne R. Presence of bicarbonate-stimulated ATPase in brush border microvillus membranes of the proximal tubule. Proc Soc Exp Biol Med 1974 ; $146: 751-3$

7. Brown D, Hirsch S, Gluck S. Localization of a proton-pumping ATPase in rat kidney. J Clin Invest $1988 ; 82$ : 2114-26.

8. De Mello Aires M, Malnic G. Sodium in renal tubular acidification kinetics. $A m \mathrm{~J}$ Physiol 1979 ; 236 : F434-F41.

9. Chantrelle B, Cogan MG, Rector FC, jr. Evidence for coupled sodium/hydrogen exchange in the rat superficial proximal convoluted tubule. Pfügers Arch 1982; 395 : 186-9.

10. Chan YL, Giebisch G. Relationship between sodium and bicarbonate transport in the rat proximal convoluted tubule. $A \mathrm{~m}$ J Physiol 1981; 240 : F222-F30.

11. Preisig PA, Ives $\mathrm{HE}$, Cragoe EJ, Jr., Alpern RJ, Rector FC, Jr. Role of the $\mathrm{Na}+/ \mathrm{H}^{+}$antiporter in rat proximal tubule bicarbonate absorption. J Clin Invest 1987 ; $80: 970-8$.

12. Burg M, Green N. Bicarbonate transport by isolated perfused rabbit proximal convoluted tubules. Am J Physiol 1977 ; 233 : F307-F14.

13. Bichara M, Paillard M, Leviel F, Gardin J-P. Hydrogen transport in rabbit kidney proximal tubules- $\mathrm{Na}$ : $\mathrm{H}$ exchange. $\mathrm{Am}$ $J$ Physiol 1980 ; 238 : F445-F51.

14. Turrini F, Sabolic I, Zimolo Z, Moewes B, Burckhardt G. Relation of ATPases in rat renal brush-border membranes to ATP-driven $\mathrm{H}^{+}$secretion. J Membrane Biol $1989 ; 107: 1-12$.

15. Sabolic I, Burckhardt G. Proton ATPase in rat renal cortical endocytotic vesicles. Biochim Biophys Acta 1988 ; 937 : 398-410. influencé par le potentiel membranaire. L'unidirectionalité de l'échange est maintenue par l'entrée du sodium dans la cellule dont la concentration cytoplasmique est maintenue basse par l'activité de la $\mathrm{Na}^{+}, \mathrm{H}^{+}$-ATPase basolatérale. Le gradient de sodium étant maintenu par l'hydrolyse de l'ATP, il s'agit d'une sécrétion de protons "secondairement active". $\mathrm{Ce}$ mécanisme a été largement étudié en partie parce qu'il prédomine dans les espèces qui se prêtent bien aux manipulations de microdissection et de microperfusion tubulaire comme le lapin.

Un second mécanisme, dont l'importance fonctionnelle est moins bien définie, implique la sécrétion proximale d'un acide organique ([5] ; revue par [1]). En effet la sécrétion de la forme protonée d'un acide organique (acide formique, acide lactique, acide $\beta$-hydroxybutyrique) suivie de la réabsorption de sa forme anionique avec du sodium (lactate, formate, $\beta$ hydroxybutyrate) produit également une sécrétion nette de protons. Encore ici, l'unidirectionalité de celleci est assurée par le gradient transmembranaire de sodium maintenu par l'activité de la $\mathrm{Na}^{+}, \mathrm{K}^{+}$-ATPase basolatérale (figure 1).

Un troisième mécanisme implique une pompe à protons localisée dans la MBB. L'énergie nécessaire au transport de $\mathrm{H}^{+}$est directement fournie par l'hydrolyse de l'ATP. Pour un ensemble de raisons reliées à des problèmes techniques (pureté des membranes isolées, spécificité des inhibiteurs etc.), la recherche sur ce mécanisme n'a commencé à progresser que récemment, en dépit de l'accumulation d'évidences indirectes plaidant pour son existence [6]. Des images d'immunolocalisation avaient montré la présence de structures compatibles avec des pompes à protons dans la $\mathrm{MBB}$ et dans des vésicules sous-membranaires [7]. De plus, un ensemble d'arguments dynamiques indirects suggéraient l'existence d'un flux de sécrétion de protons indépendant de l'activité de la $\mathrm{Na}^{+}$, $\mathrm{K}^{+}$-ATPase dans le tubule proximal de rat. L'inhibition de celle-ci par la ouabaïne, limitant secondairement l'activité de l'échangeur $\mathrm{Na}^{+} / \mathrm{H}^{+}$, ne supprimait que $30 \%$ du transport du bicarbonate dans le tube proximal microperfusé de rat $[8,9]$. De même, le remplacement du sodium dans le liquide luminal d'un tubule perfusé, ou la présence d'amiloride, n'abolissait pas complètement le flux de réabsorption de bicarbonate [10, 11]. Chez le lapin cependant, une fraction plus faible du flux de réabsorption de bicarbonate ou de la sécrétion de protons était indépendante du sodium, suggérant une différence entre ces espèces $[5,12,13]$.

Des étude réalisées sur vésicules membranaires obtenues de $\mathrm{MBB}$ de rat ainsi que sur vésicules endocytotiques sous-membranaires ont permis de démontrer l'existence d'une activité ATPasique de type $\mathrm{H}^{+}$-ATPase dans ces membranes $[14,15]$. L'équivalent physiologique de cette activité enzymatique correspondait à une pompe à protons de type vacuolaire. Ces études avaient permis de définir l'affinité de la pompe pour les nucléotides triphosphatés et son profil d'inhibition par différents inhibiteurs de spécificité variée $[14,15]$. Ces travaux établissaient donc la présence de ce mécanisme de transport de protons sur la $\mathrm{MBB}$ du cortex rénal de rat mais ne définissaient pas l'importance relative des trois mécanismes de sécrétion de protons dans le tubule proximal intact.

Afin de quantifier l'importance relative de la $\mathrm{H}^{+}$-ATPase pour la sécrétion de protons, nous avons utilisé un animal carnivore, le chien. Nous nous sommes penchés sur l'existence, la régulation et le coût métabolique de la sécrétion de protons par cette pompe proximale. Nos expériences démontrent que dans des conditions normales, et plus encore dans certaines conditions pathologiques (acidose et alcalose), ce mécanisme est probablement responsable d'une fraction significative de la réabsorption proximale des bicarbonates chez cette espèce.

\section{La pompe à protons entre la membrane et l'endosome}

Les pompes à protons présentes dans la MBB sont formées d'un segment intramembranaire lipophile, correspondant à un canal à protons, associé à un complexe hydrophile globulaire cytoplasmique sur lequel on 


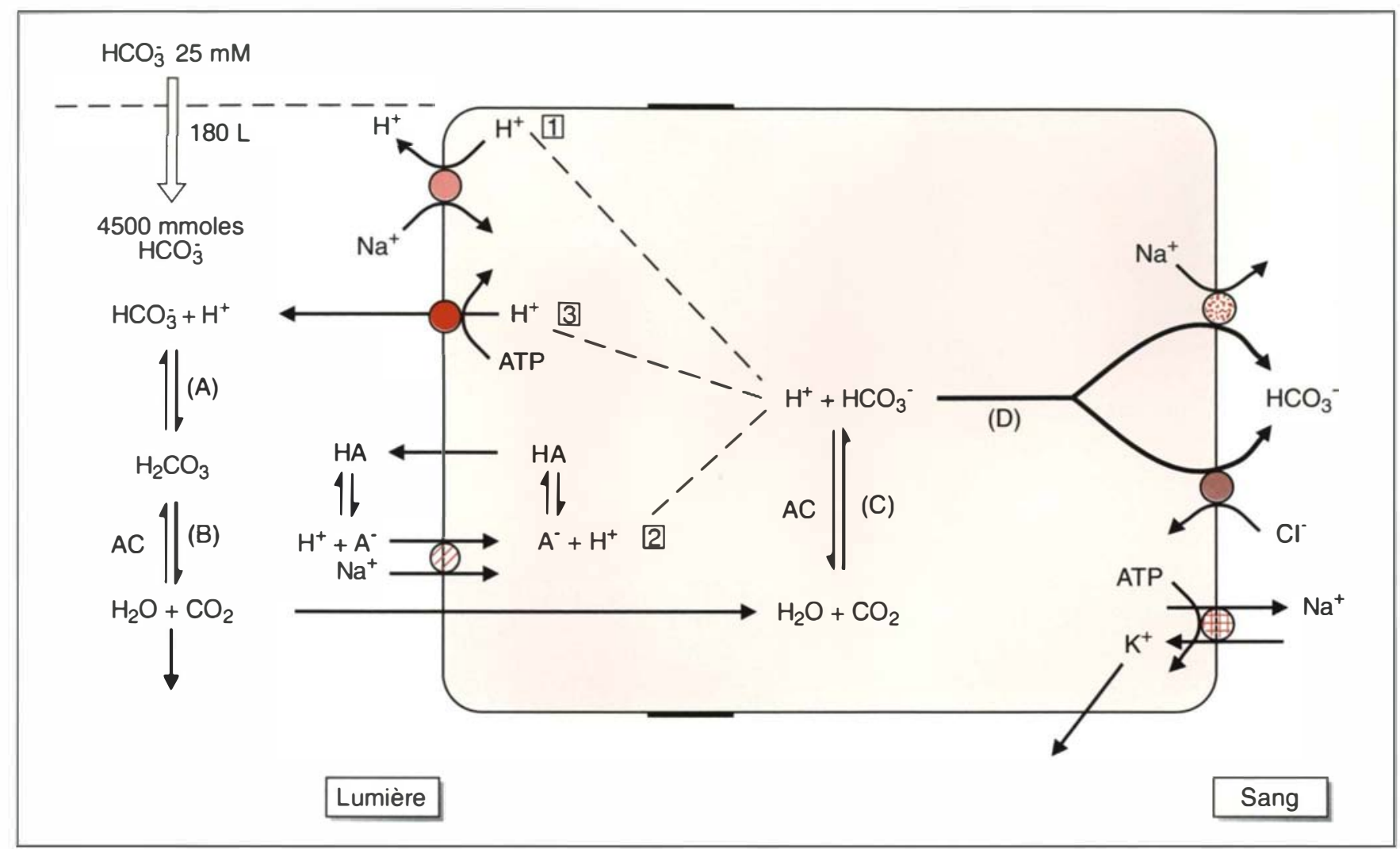

Figure 1. Mécanismes impliqués dans la sécrétion proximale de protons. [1]: échangeur $\mathrm{Na}+/ H+f o n c t i o n-$ nellement couplé à la $\mathrm{Na}^{+}, \mathrm{K}^{+}-\mathrm{ATPase}$ basolatérale, [2] : sécrétion de métabolites protonés (HA) suivie de la réabsorption de leurs formes anioniques (A-) avec le sodium et [3] : $H^{+}-A T P a s e$ (pompe à protons). La sécrétion de protons par ces trois mécanismes assure la réabsorption des bicarbonates. A) Les bicarbonates sont d'abord transformés par la sécrétion luminale de protons en acide carbonique $\left(\mathrm{H}_{2} \mathrm{CO}_{3}\right)$. B) Sous l'effet d'une anhydrase carbonique membranaire (AC), cet acide carbonique se dissocie dans la lumière tubulaire en eau et en $\mathrm{CO}_{2}$. C) Celui-ci diffuse dans le compartiment cytoplasmique où une anhydrase carbonique cellulaire catalyse la resynthèse d'acide carbonique qui se dissocie, régénérant dans la cellule le proton précédemment sécrété et libérant un ion bicarbonate. D) Les ions bicarbonates ainsi régénérés sont expulsés de la cellule au niveau de la membrane basolatérale au moyen de mécanismes impliquant soit un échange $\mathrm{Cl}-\mathrm{HCO}_{3^{-}}$soit un cotransporteur $\mathrm{Na}+3 \mathrm{HCO}_{3^{-}}$.

retrouve les sites de liaison de l'ATP (revues par [16] et [17]). L'énergie libérée par l'hydrolyse de l'ATP induit un changement de conformation conduisant à une sécrétion de protons. Lorsque I'on prépare des vésicules de $\mathrm{MBB}$, les feuillets membranaires se présentent sous plusieurs configurations qui sont présentées au bas de la figure 2. La majorité des membranes se referment spontanément selon leur polarité naturelle, enfermant dans la lumière de la vésicule les sites de liaison de l'ATP de la pompe à protons. On ne peut donc révéler l'activité ATPasique spécifique de ces unités sans solubiliser auparavant les membranes pour permettre l'accès de l'ATP à l'enzyme. Par ail- leurs, ces pompes à protons existent également sous une forme de réserve dans des endosomes sousmembranaires : ceux-ci exposent spontanément le complexe hydrophile de leurs pompes à l'ATP cellulaire. La préparation de MBB est toujours plus ou moins contaminée d'endosomes. On s'attend donc à mesurer d'abord l'activité ATPasique spontanée de ces endosomes (ainsi que celle de feuillets de MBB non refermés et/ou de vésicules inversées) et à ne révéler l'activité ATPasique intravésiculaire qu'après solubilisation des membranes par un détergent.

Le panneau de gauche de la figure 2 présente l'hydrolyse de l'ATP mesurée sur une suspension vésiculaire de
MBB de chien. L'activité initiale est attribuable aux endosomes et aux membranes non refermées en vésicles étanches qui exposent spontanément leurs sites de fixation de l'ATP au milieu extravésiculaire. Cette activité augmente de cinq fois à la suite de la solubilisation des membranes et de l'ouverture des vésicules de MBB par le déoxycholate. Il s'agit d'une activité sensible au $\mathrm{N}$-éthylmaléimide (NEM) comme à la bafilomycine $A^{1}$, deux inhibiteurs des $\mathrm{H}^{+}$-ATPases vacuolaires (revue par [16] ; [15]). Ce profil d'inhibition démontre bien la nature vacuolaire de cet enzyme, et la stimulation de l'activité observée après solubilisation des membranes confirme que la 


\section{RÉFÉRENCES}

16. Forgac M. Structure and function of vacuolar class of ATP-driven proton pumps. Physiol Rev 1989 ; 69 : 765-796.

17. Stone DK, Crider BP, Xie X-S. Structure of vacuolar proton pumps. Sem in Nephrology $1990 ; 10: 159-65$.

18. Bowman EJ, Siebers A, Attendorf K Bafilomycins : A class of inhibitors of membrane ATPases from microorganisms, animal cells, and plant cells. Proc Natl Acad Sci USA 1988 ; 85 : 7972-6.

19. Noël J, Laprade R, Vinay P. The bafilomycin (Baf)-sensitive $\mathrm{H}^{+}$-ATPase requires $30 \%$ of the cell ATP turnover in dog renal proximal tubules (PT), but $10 \%$ in rabbit PT. FASEB J 1992 ; 6 : A 1460.

20. Alberti KGMM, Cuthbert C. The hydrogen ion in normal metabolism : a review. In : Ciba Foundation Symposium. ed. Metabolic Acidosis. Pitman Books Ltd. London : $1982 ; 1-19$.

21. Simon BJ, Burckhardt G. Characterization of inside-out oriented $\mathrm{H}^{+}$-ATPases in cholate-pretreated renal brush-border membrane vesicles. J Membrane Biol 1990 ; $117: 141-51$.

22. Noël J, Laprade R, Burckhardt G, Gougoux A, Vinay P. A brush border membrane-bound $\mathrm{H}^{+}$-ATPase from the dog proximal tubule. Cell Physiol Biochem $1992 ; 2$ : 18-36.

23. Noël J, Bellemare F, Thiéry G, Burckhardt $G$, Laprade R, Vinay P. Effect of cholate treatment on dog brush-border membrane (BBM) proteins including the

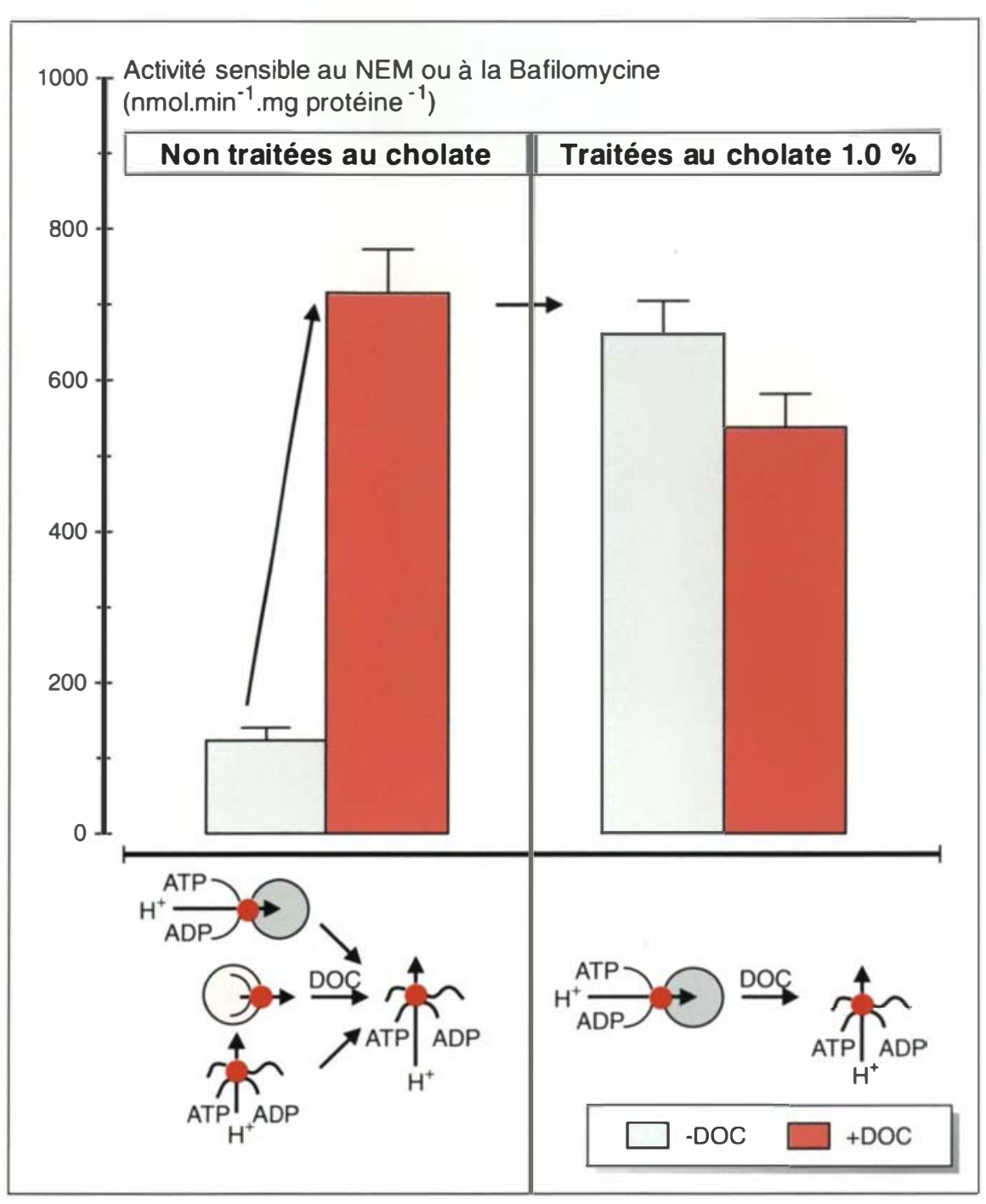

Figure 2. Effet de la solubilisation des membranes par le déoxycholate $0.1 \%$ (DOC) sur l'activité H+-ATPasique sensible au NEM lou à la bafilomycinel de vésicules de MBB de chien traitées ou non traitées au cholate de sodium $1 \%$. La conformation des éléments membranaires avant et après solubilisation est représentée au bas de chaque panneau. La pompe à protons est représentée par un cercle fermé doté d'un trident représentant le site de liaison de l'ATP, la génération d'ADP et la fixation d'un proton. Les vésicules acidifiées sont présentées en gris. (Figure adaptée de [22]).

majorité des $\mathrm{H}+$-ATPases présentaient un site intravésiculaire de liaison pour l'ATP, en accord avec une polarité naturelle permettant la sécrétion de protons dans la lumière tubulaire. La préparation de MBB utilisée est donc peu contaminée par des endosomes et présente néanmoins une activité $\mathrm{H}^{+}$-ATPase importante.

L'enrichissement en $\mathrm{H}^{+}$-ATPase mesuré sur ces vésicules est comparable à l'enrichissement observé pour d'autres marqueurs connus de cette membrane, telles la $\boldsymbol{\gamma}$-glutamyltranspeptidase $(\gamma-G T)$ et la phosphatase alcaline (figure 3). La copurification de ces trois protéines suggère fortement leur coexistence dans la MBB. Par ailleurs, on note un appauvrissement en marqueurs de la membrane basolatérale $\left(\mathrm{Na}^{+}, \mathrm{K}^{+}\right.$-ATPase $)$et mitochondriale $\left(\mathrm{H}^{+}\right.$-ATPase sensible à l'oligomycine et glutamate déshydrogénase). Nous trouvons donc une $\mathrm{H}^{+}$-ATPase vacuolaire sur la $\mathrm{MBB}$ du tubule proximal de chien. 


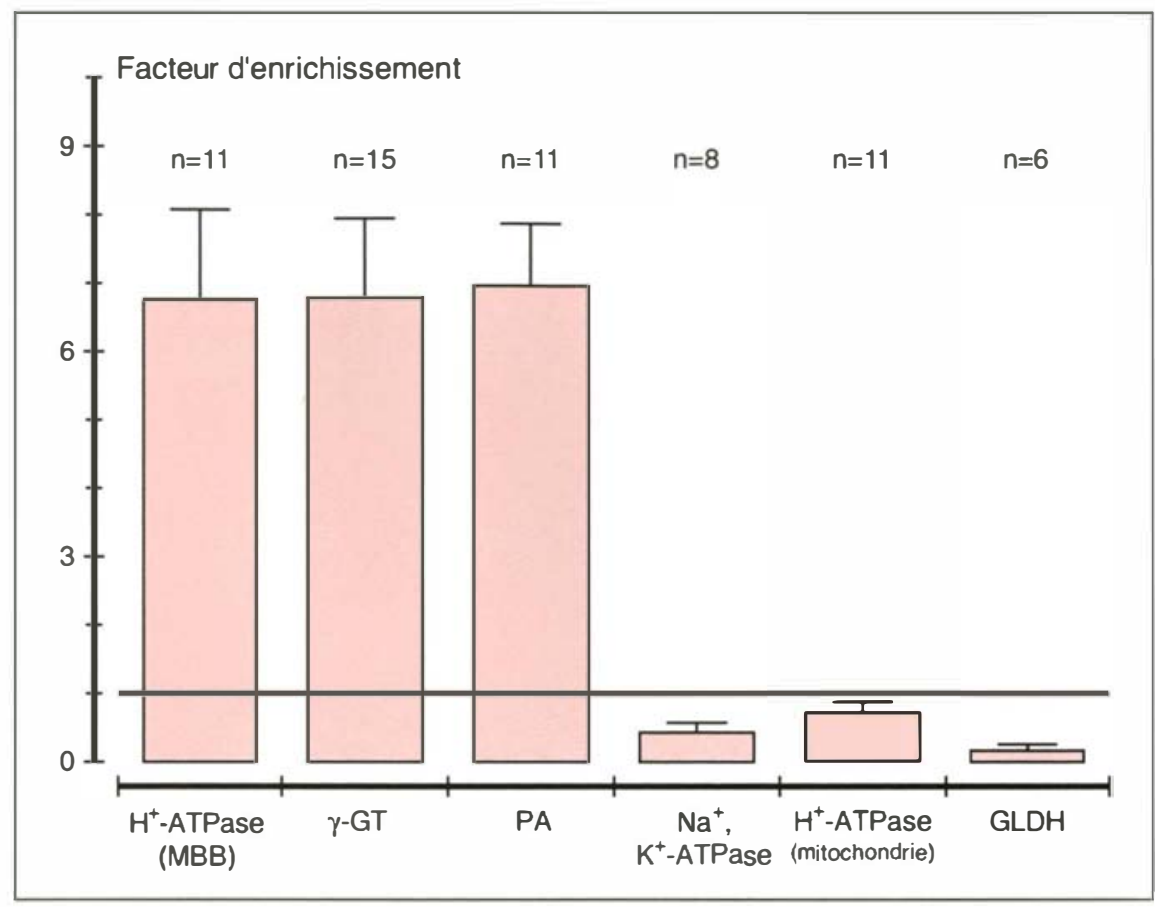

Figure 3. Enrichissement de l'activité de marqueurs membranaires dans des préparations de MBB par comparaison avec I'homogénat de cortex rénal de départ. $\gamma$-GT : $\gamma$-glutamyltranspeptidase, PA : phosphatase alcaline ; GLDH : glutamate déshydrogénase.

La figure 4 présente les caractéristiques d'inhibition de cette ATPase. Son insensibilité vis-à-vis de l'oligomycine exclue une activité d'origine mitochondriale. Elle est aussi insensible au vanadate; il ne s'agit donc pas d'une protéine autophosphorylée (enzyme de type E1-E2 comme la $\mathrm{H}^{+}, \mathrm{K}^{+}$-ATPase, la $\mathrm{Na}^{+}$, $\mathrm{K}^{+}$-ATPase ou la $\mathrm{Ca}^{++}$-ATPase). Elle est inhibée par le N,N'dicyclohexylcarbodiimide (DCCD), le NEM, le chlorure de 7-chloro-4-nitrobenz-2-oxa-1,3-diazole (NBD-Cl), trois inhibiteurs puissants mais relativement peu spécifiques des $\mathrm{H}^{+}$-ATPases de type vacuolaire. Elle est enfin remarquablement inhibée par de faibles concentrations de bafilomycine $A_{1}$, qui n'affecte ni l'activité de la $\mathrm{Na}^{+}, \mathrm{K}^{+}$-ATPase ni celle de l'ATP synthétase, et qui ne modifie pas la concentration cellulaire d'ATP de tubules proximaux isolés $[18,19]$ (figure 4). La bafilomycine $A_{1}$ apparaît donc un outil idéal pour évaluer le rôle de la $\mathrm{H}^{+}$-ATPase dans les tubules intacts (voir ci-dessous).
Burkhardt, alors du Max Planck Institute für Biophysik, Francfort [21]. Ces auteurs ont en effet décrit une manœuvre permettant d'inverser la polarité de la pompe dans les $\mathrm{MBB}$ au moyen d'un court traitement au cholate de sodium suivi d'une dialyse prolongée. Le panneau de droite de la figure 2 montre qu'après ce traitement, l'activité ATPasique totale des vésicules de $\mathrm{MBB}$ de chien non solubilisées est maximale et comparable, qu'on la mesure avant ou après solubilisation des membranes avec un détergent puissant : le déoxycholate. Cela indique que la solubilisation n'est plus requise pour exposer les sites de liaison à l'ATP de la pompe à $\mathrm{H}^{+}$au milieu extravésiculaire. Le cholate n'a pas solubilisé les membranes puisque l'ajout d'ATP entraîne une acidification intravésiculaire intense mesurée en fluorescence par l'accumulation d'orange d'acridine, indiquant la présence de compartiments relativement étanches i.e. de vésicules refermées non solubilisées.

La figure 5 présente le changement de $\mathrm{pH}$ intravésiculaire produit par l'addition d'ATP dans le milieu d'incubation de vésicules de MBB traitées ou non traitées au cholate. Ce milieu contenait un système régénérateur d'ATP afin de maintenir basse et constante la concentration d'ADP autour de la pompe [22]. Une acidification importante n'est mesurée qu'avec les vésicules traitées au cholate. Celle-ci est complètement prévenue ou renversée par l'addition de NEM ou de bafilomycine. Le gradient de protons est aboli par l'addition d'un échangeur $\mathrm{K}+/ \mathrm{H}^{+}$, la nigéricine, qui s'insère dans la membrane et dissipe toute différence de concentration de protons. La figure 5 confirme donc que l'acidification des vésicules est bien dépendante de l'hydrolyse de l'ATP extravésiculaire, que l'orientation de la pompe a été inversée par le traitement au cholate et que les membranes sont refermées en structures relativement étanches aux protons. L'inhibition importante et irréversible de l'échangeur $\mathrm{Na}^{+} / \mathrm{H}^{+}$par le traitement au cholate de même que l'absence de $\mathrm{Na}^{+}$dans le milieu favorisent cette étanchéité [23]. La $\mathrm{H}^{+}$-ATPase de la MBB du rein du chien est donc capable d'effectuer un transport net de protons. 


\section{RÉFÉRENCES}

24. Vinay P, Noël J, Tejedor A, Gougoux A. Le métabolisme cellulaire in vitro : effets métaboliques méconnus. $\mathrm{m} / \mathrm{s} 1990 ; 6$ : 268-78.

25. Wang Z-Q, Gluck S. Isolation and properties of bovine kidney brush border vacuolar $\mathrm{H}^{+}$-ATPase. A proton pump with enzymatic and structural differences from kidney microsomal $\mathrm{H}^{+}$-ATPase. J Biol Chen 1990 ; 265 : 21957-65.

26. Noël J, Tejedor A, Vinay P, Laprade R. Substrate-induced modulation of ATP turnover in dog and rabbit proximal tubules. J Membrane Biol 1992 ; 128 : 205-18.

27. Ullrich KJ, Capasso G, Rumrich G, Papavassiliou F, Klöss S. Coupling between proximal tubular transport processes. Studies with ouabain, SITS and $\mathrm{HCO} 3$ - free solutions. Pflügers Arch. 1977 ; 368 : 245-52.

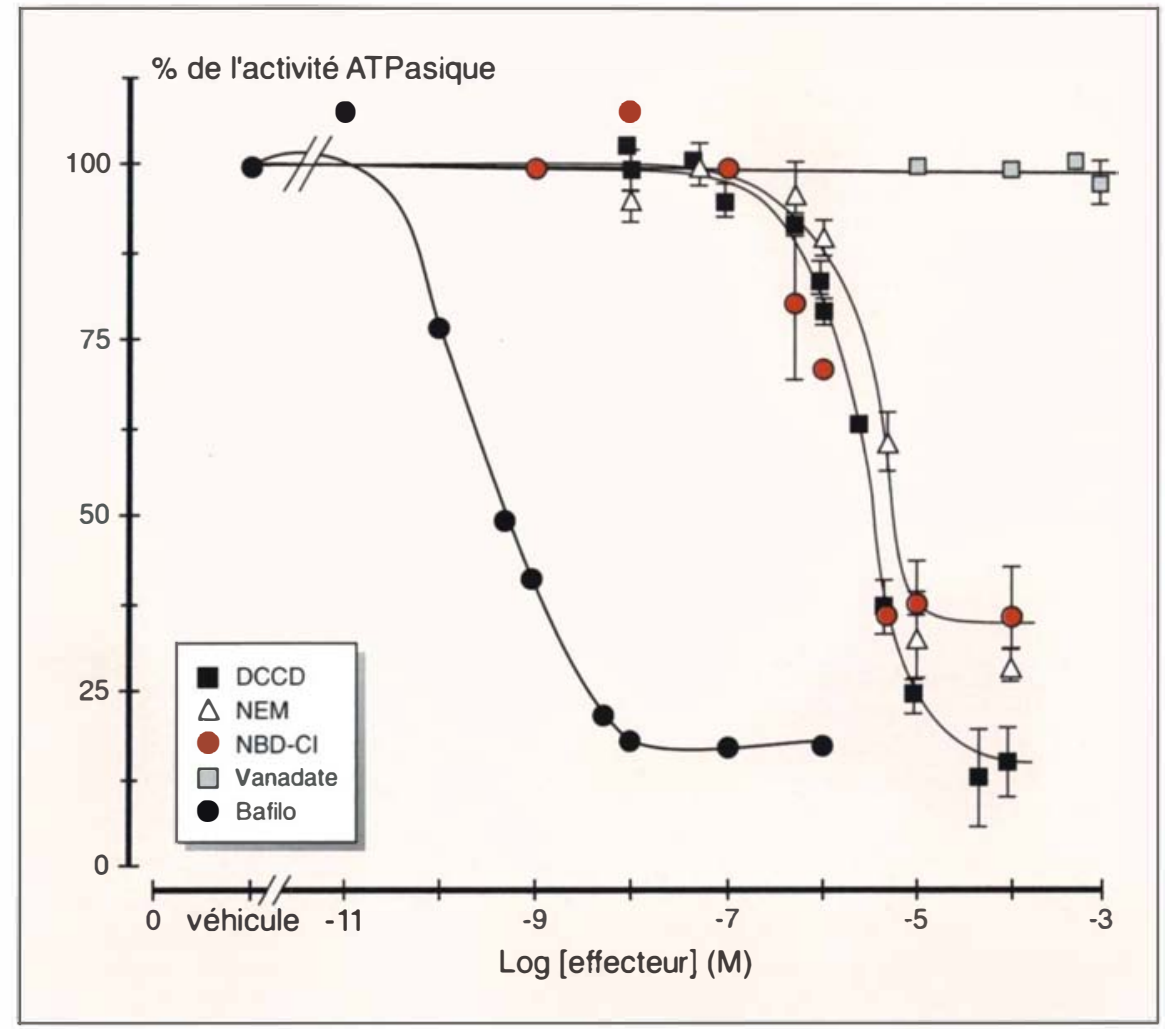

Figure 4. Courbe dose-effet montrant la réponse de l'activité ATPasique, insensible à l'oligomycine et à la ouabaïne, à différents inhibiteurs connus des ATPases ionomotrices. Le $1_{50}$ apparent déterminé respectivement pour le DCCD est de $2.6 \times 10^{-6} \mathrm{M}(n=4)$; pour le NEM de $4.5 \times 10^{-6}$ $M(n=3)$; pour le NBD-Cl de $1.0 \times 10^{-6} \mathrm{M}(n=2)$; pour la bafilomycine de $2 \times 10^{10} \mathrm{M}(n=1)$. L'activité est exprimée en pourcentage de l'activité maximale mesurée sur les membranes solubilisées en présence du véhicule de ces agents (eau, éthanol, DMSO). Le fait que les courbes sigmoïdales d'inhibition ne rejoignent pas toutes le même niveau peut s'expliquer en partie en raison de l'effet variable de ces inhibiteurs sur les activités ectoATPasiques insensibles au déoxycholate, également présentes sur ces membranes [14, 22], et en partie par une variabilité saisonnière d'activité ATPasique ladaptée de [22]).

Notons que le traitement au cho3late n'entraîne pas une inversion de la polarité des vésicules. En effet, les ectoenzymes $\gamma$-GT, phosphatase alcaline et aminopeptidase neutre, dont l'activité est mesurée au moyen de substrats artificiels peu perméants, demeurent entièrement exposés au milieu d'incubation avant comme après le traitement au cholate. De plus, aucune activité additionnelle n'est révélée à la suite de la solubilisation de ces membranes par le déoxycholate [23].

\section{Une sécrétion de protons qui coûte cher}

Cette pompe joue-t-elle un rôle signi- ficatif pour la sécrétion de protons par la cellule proximale intacte? Afin de répondre à cette question, nous avons utilisé des suspensions de tubules proximaux de chien. Sur ces suspensions purifiées, il est possible de quantifier la synthèse cellulaire d'ATP i.e. le versant métabolique du travail cellulaire, en suivant la consommation d'oxygène et en déterminant la fraction de cette respiration qui mène directement à la synthèse de l'ATP (respiration phosphorylante ; [24]). Cette quantité correspond à la respiration inhibée par l'inhibiteur spécifique de l'ATPsynthétase mitochondriale : l'oligomycine.

La figure 6 montre qu'environ $70 \%$ 
de la respiration cellulaire de tubules proximaux est inhibée par l'oligomycine $(100 \%$ de la respiration phosphorylante), $30 \%$ de la respiration étant attribuable aux processus assurant une entrée de $\mathrm{H}^{+}$dans la mitochondrie par des voies alternatives à l'ATP synthétase [24]. L'oli- gomycine réduit à zéro la concentration cellulaire d'ATP, indiquant le caractère non phosphorylant de la respiration résiduelle et l'absence de synthèse cytoplasmique significative d'ATP dans ces cellules proximales peu glycolytiques.

Il devient possible d'estimer, au

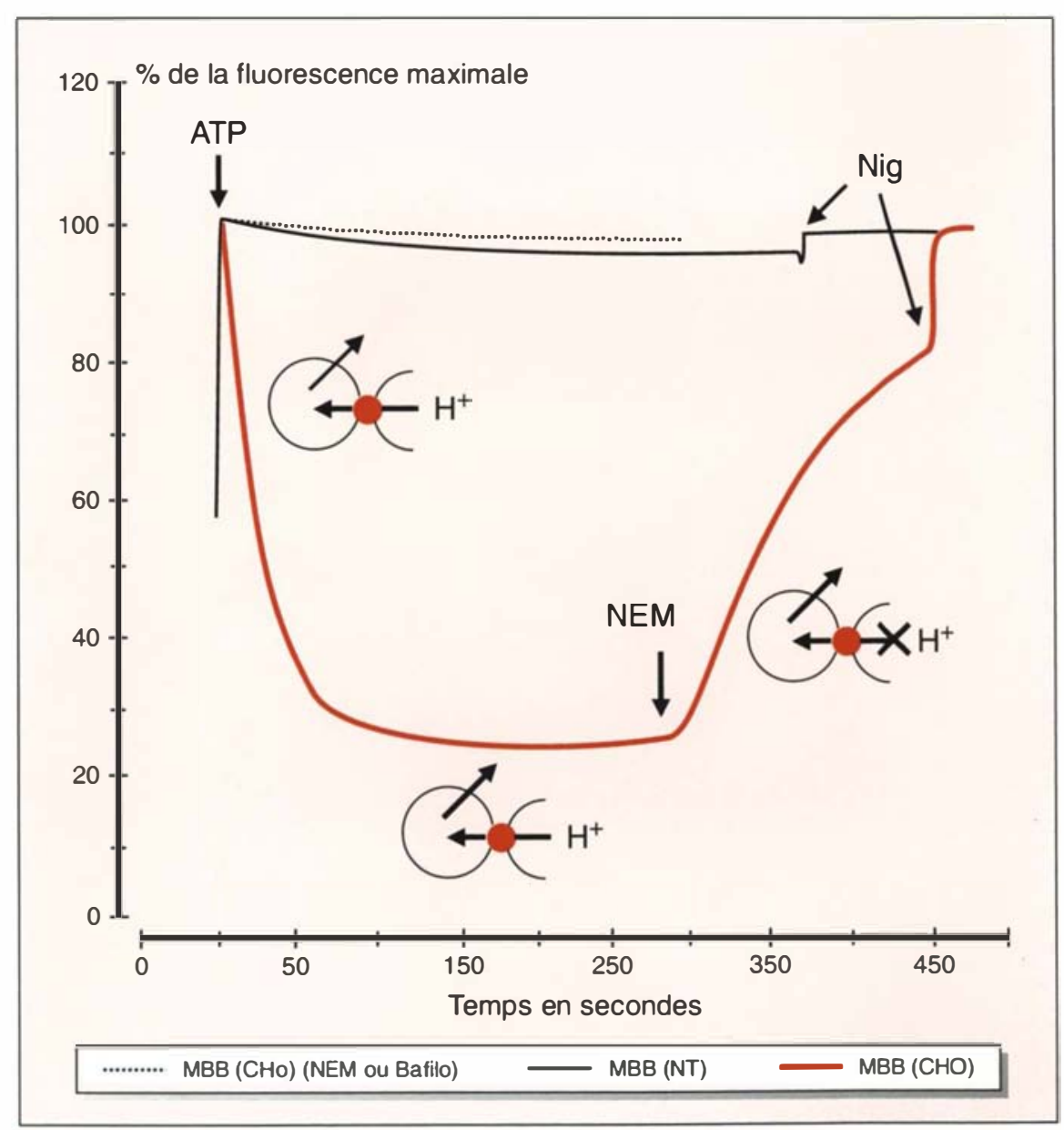

Figure 5. Acidification intravésiculaire de vésicules de MBB de chien traitées (CHO) ou non traitées (NT) au cholate (400 $\mu \mathrm{g}$ protéines) induite par I'ajout d'ATP. Le tracé inférieur obtenu avec les $\mathrm{MBB}$ (CHO) peut être divisé en trois grandes parties illustrées par des représentations de l'état des vésicules: 1) une diminution de la fluorescence traduisant une acidification de la vésicule i.e. une entrée de $\mathrm{H}^{+}$supérieure à la sortie passive de $\mathrm{H}^{+}$2) un plateau indiquant une entrée de $\mathrm{H}^{+}$équivalente à la sortie passive de $\mathrm{H}^{+}$et 3 ) une augmentation de la fluorescence traduisant une alcalinisation intravésiculaire progressive i.e. une sortie de $\mathrm{H}^{+}$excédant l'entrée active de $\mathrm{H}^{+}$linhibition de la $\mathrm{H}^{+}$-ATPase induite par l'addition aiguë de NEM). Le tracé intermédiaire montre l'acidification modeste observée avec les vésicules natives (NT). Le tracé supérieur montre l'effet d'un prétraitement $(10 \mathrm{~min})$ des MBB (CHO) au NEM $10^{-3} \mathrm{M}$ (NEM) ou à la bafilomycine $A, 10^{-6} \mathrm{M}$ (Bafilo, $0.4 \mathrm{nmoles} / \mathrm{mg}$ protéine), deux inhibiteurs de la pompe à protons. La nigéricine (Nig) est utilisée pour abolir la différence de $\mathrm{pH}$ créé de part et d'autre des vésicules.

$\mathrm{m} / \mathrm{s} n^{\circ} 5$ vol. 9, mai 93 moyen d'inhibiteurs spécifiques, la contribution relative des principales ATPases cellulaires à l'hydrolyse et à la synthèse de l'ATP. Ces deux grandeurs étant égales par définition, l'effet d'inhibiteurs spécifiques des ATPases sur la consommation d'oxygène couplée fournit cette information. La colonne centrale de la figure 7 montre que $44 \%$ de la respiration phosphorylante de tubules proximaux de chien est inhibée par la ouabaïne, indiquant le coût métabolique du transport actif de sodium par la $\mathrm{Na}^{+}, \mathrm{K}^{+}$-ATPase. Cela inclut le transport de sodium lié à la sécrétion de protons par l'échangeur $\mathrm{Na}+/ \mathrm{H}^{+}$ ou à la réabsorption de la forme anionique de certains acides organiques. Une autre fraction de $44 \%$ est inhibée par la bafilomycine $A_{1}$, un inhibiteur spécifique de la $\mathrm{H}^{+}$-ATPase. Donc $88 \%$ de la synthèse proximale d'ATP est attribuable à l'activité de ces deux ATPases chez le chien; toutes les autres fonctions d'hydrolyse de l'ATP de la cellule ne requérant que le $12 \%$ résiduel.

Ainsi, l'hydrolyse d'ATP requise pour soutenir l'activité de la pompe à protons est donc au moins aussi grande que celle utilisée pour maintenir l'activité de la pompe à sodium dans une suspension de tubules proximaux. Cela suggère que la sécrétion de protons par cette pompe est une opération coûteuse sur le plan énergétique. Elle est même probablement plus coûteuse que la réabsorption sodée étant donné que la concentration de sodium est six fois plus élevée que la concentration de bicarbonate dans le fluide tubulaire. En effet, même si le sodium n'est réabsorbé qu'à 70-80 \% dans le tubule proximal (le bicarbonate étant entièrement réabsorbé), le nombre de molécules de sodium transportées dépasse largement le nombre de molécules de bicarbonate transportées. Ces études ont été réalisées avec des tubules proximaux de chien fraîchement isolés et incubés dans un milieu extracellulaire artificiel à $\mathrm{pH}$ 7.4 et contenant du bicarbonate à $25 \mathrm{mM}$ et du sodium à $150 \mathrm{mM}$. La situation particulière des tubules étudiés in vitro, en absence de la limitation imposée par la filtration glomérulaire (fixant la disponibilité de 
$\mathrm{Na}^{+}$et de $\mathrm{HCO}_{3}^{-}$, explique peutêtre ce résultat [24].

\section{Caractéristiques de la pompe à protons}

Le Tableau I résume les caractéristiques fonctionnelles de la pompe à protons de la MBB. Chez le chien, celle-ci est électrogénique et son activité maximale est obtenue à un $\mathrm{pH}$ entre 7.3 et 7.5 [22]. Son $\mathrm{Km}$ pour l'ATP mesuré en mode de transport de $\mathrm{H}^{+}$est de $150 \mu \mathrm{M}[22]$. Simon et Burckhardt avaient trouvé une valeur de $90 \mu \mathrm{M}$ pour la $\mathrm{H}^{+}$-ATPase de MBB de porc [21] et Wang et Gluck $340 \mu \mathrm{M}$ pour l'enzyme purifié du cortex rénal de bovin [25]. Le Ki pour l'ADP est de $24 \mu \mathrm{M}$ [21], suggérant une sensibilité " exquise " de cette activité aux variations d'ADP cellulaire. L'activité de la $\mathrm{H}^{+}$-ATPase n'est pas dépendante de la présence de chlore lorsque la compensation des charges est assurée [22].

\section{Une importante différence entre les espèces}

Les mesures de consommation d'oxygène de tubules proximaux de chien suggèrent que la pompe à protons soit un mécanisme majeur de réabsorption des ions bicarbonates chez

\section{RÉFÉRENCES}

28. Schwartz GJ, Al-Awqati Q. Carbon dioxide causes exocytosis of vesicles containing $\mathrm{H}^{+}$pumps in isolated perfused proxi$\mathrm{mal}$ and collecting tubules. J Clin Invest $1985 ; 75: 1638-44$

29. Brown D, Sabolic I, Gluck S. Colchicine-induced redistribution of proton pumps in kidney epithelial cells. Kidney Int 1991; 40 (Suppl. 33) : S-79-S-83.

30. Brown D, Gluck S, Hartwig J. Structure of the novel membrane-coating material in proton-secreting epithelial cells and identification as an $\mathrm{H}^{+}$-ATPase. $J$ Cell Biol $1987 ; 105: 1637-48$.

31. Schwartz GJ, Al-Awqati Q. Regulation of transepithelial $\mathrm{H}+$ transport by exocytosis and endocytosis. Ann Rev Physiol 1986 ; $48: 153-61$.

32. Aronson PS, Nee J, Suhm MA. Modifier role of internal $\mathrm{H}^{+}$in activating the $\mathrm{Na}^{+} \cdot \mathrm{H}^{+}$exchanger in renal microvillus membrane vesicles. Nature 1982; 299 : 161-3. cette espèce carnivore. Rappelons que les études antérieures mesurant la sécrétion proximale de protons chez le lapin impliquaient l'échangeur

$\mathrm{Na}^{+} / \mathrm{H}^{+}$de façon prépondérante et non la $\mathrm{H}^{+}$-ATPase. Il nous a semblé important de vérifier si la $\mathrm{H}^{+}$-ATPase était présente chez le

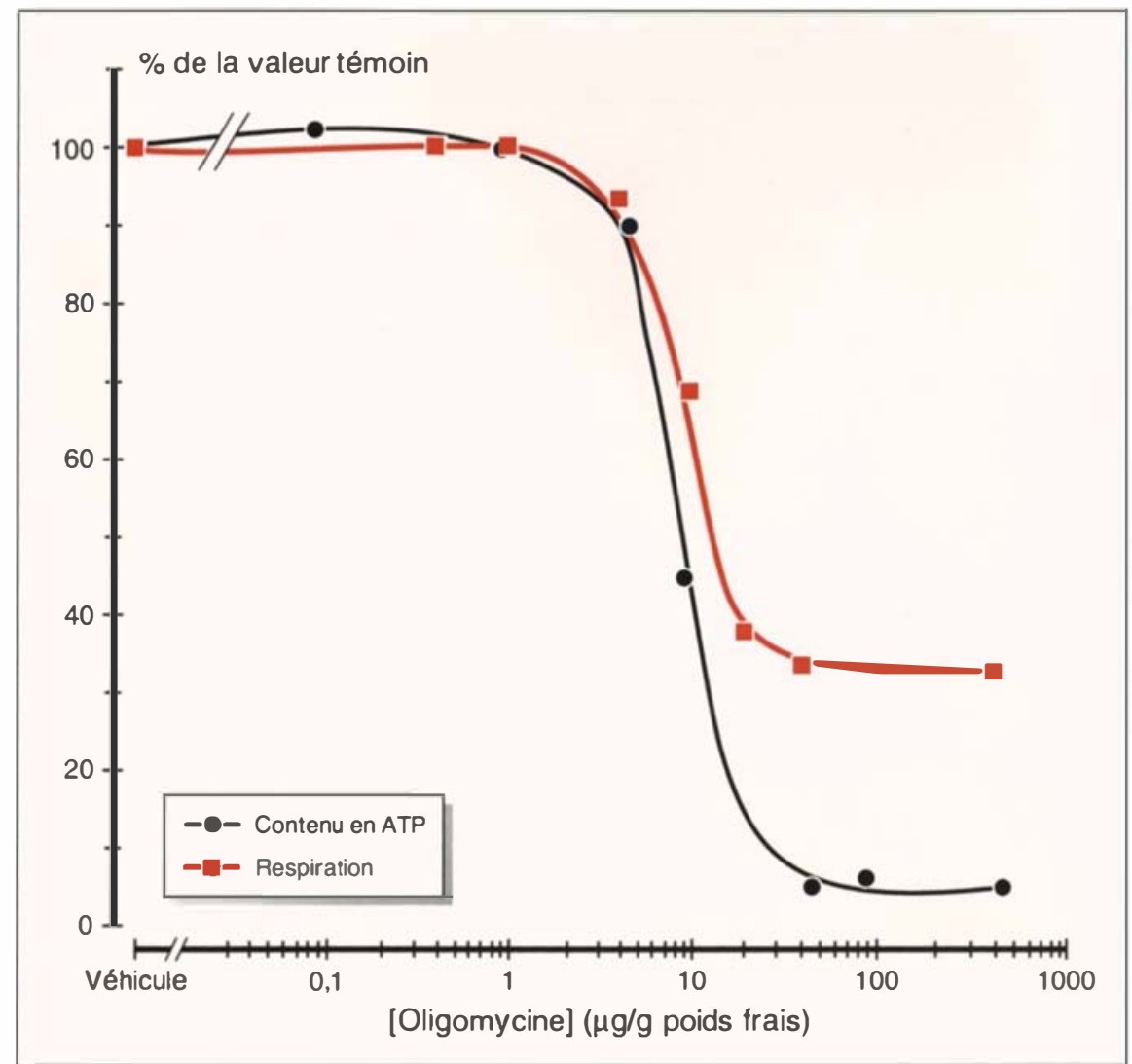

Figure 6. Effets de l'oligomycine sur la respiration cellulaire et sur le contenu en ATP de tubules proximaux de chien. Les mesures ont été effectuées en présence des substrats exogènes suivants : lactate : pyruvate (10: $1 \mathrm{mM}$ ) et glutamine : glutamate (10: $1 \mathrm{mM}$ ) (tirée de [26]).

\section{Tableau I}

\section{CARACTÉRISTIQUES DE LA POMPE A PROTONS DE LA MBB}

Activée par l'ATP

Inhibée par le DCCD, le NEM, le NBD-C1, la Bafilomycine $A_{1}$

Non inhibée par l'oligomycine, la ouabaïne, le vanadate

Non dépendante du chlore

Électrogénique (inhibée par un potentiel membranaire positif)

Activité optimale à $\mathrm{pH}$ 7.3-7.5

Affinité élevée pour l'ATP: $\mathrm{Km}=90-340 \mu \mathrm{M}$

Inhibée par l'ADP: Ki $=24 \mu \mathrm{M}$

Régulée par les paramètres acidobasiques intracellulaires 
lapin et si elle y requérait une fraction significative de la synthèse proximale d'ATP. Les mesures de l'activité ATPasique sensible au NEM de $\mathrm{MBB}$ de lapin nous ont révélé une activité 4 à 5 fois plus faible que chez le chien [26]. La figure 7 montre que seulement $5 \%$ de la respiration phosphorylante est requise pour soutenir l'activité d'une pompe à protons sensible à la bafilomycine $\mathrm{A}_{1}$ chez le lapin. Il est donc probable que chez cette espèce herbivore, la contribution respective des mécanismes de sécrétion de protons s'agence différemment que chez le chien. Chez le hamster, où l'importance de l'échangeur $\mathrm{Na}^{+} / \mathrm{H}^{+}$avait été remise en question par les observations de Ullrich et collaborateurs [27], nous trouvons, comme chez le lapin, une faible contribution de la $\mathrm{H}^{+}$-ATPase à l'utilisation totale d'ATP (figure 7). Notons que les différences observées entre les espèces ne peuvent être attribuées aux conditions expérimentales utilisées pour chaque espèce (type de substrat exogène, etc.).

Ces résultats réconcilient entre elles les observations antérieures suggérant une activité prépondérante de l'échangeur $\mathrm{Na}^{+} / \mathrm{H}^{+}$pour la réabsorption des bicarbonates chez ces espèces et démontrent clairement que l'extrapolation du lapin au chien est impossible. L'extrapolation à l'homme doit, par conséquent, être vérifiée expérimentalement.

\section{Régulation de la pompe à protons}

La présence d'une pompe à protons dans la $\mathrm{MBB}$, et sa distribution sur la membrane plasmique ou sur les structures vésiculaires sousmembranaires, de même que les caractéristiques enzymatiques et biochimiques de cette pompe, permettent de proposer au moins sept mécanismes différents susceptibles d'assurer une régulation de la sécrétion de protons par la $\mathrm{H}^{+}$-ATPase.

1) On peut envisager une régulation de l'activité par un changement de la densité de l'affichage des pompes à protons sur la $\mathrm{MBB}$. Celle-ci résulte d'un équilibre dynamique d'insertion et de retrait entre les vésicules sousmembranaires et la membrane cellulaire luminale. Le système microtu$m / s n^{\circ} 5$ vol. 9, mai 93 bulaire participerait à ces échanges de protéines entre les deux membranes $[28,29]$. Une élégante indication optique de ce processus a été fournie par Brown et collaborateurs dans les cellules distales [30] et proximales [29]. En effet, dans une cellule de type $\alpha$ de vessie de crapaud, ils ont identifié une vésicule endocytotique recouverte de structures globulaires correspondant aux complexes hydrophiles immunologiquement actifs des pompes à protons. Cette vésicule était associée à un filament d'actine [30]. La régulation de l'affichage des unités $\mathrm{H}^{+}$-ATPasiques pourrait ainsi

être dictée par les paramètres acidobasiques cellulaires (revues par [1, 2]), incluant la pression locale de $\mathrm{CO}_{2}[28]$. Les modifications de la concentration intracellulaire en calcium pourraient aussi réguler le processus de fusion des endosomes à la membrane plasmique $[28,31]$.

2) L'activité des unités ATPasiques déjà insérées dans la membrane pourrait également être modulée par les paramètres acidobasiques de la cellule. Il s'agirait, par exemple, d'une régulation directe par le $\mathrm{pH}$ environnant la pompe. Ainsi, nous avons montré que des petites variations de

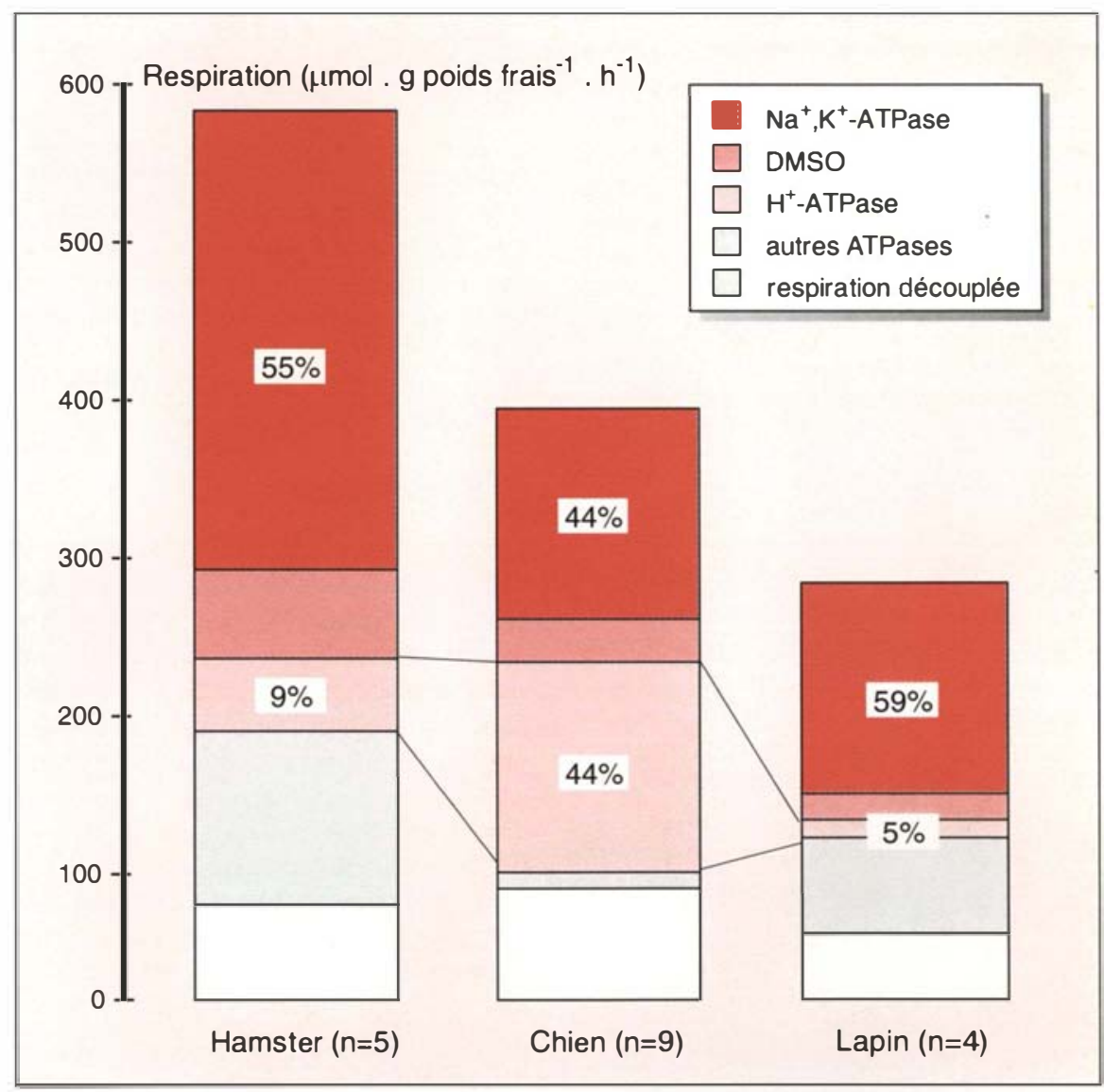

Figure 7. Identification des différentes composantes respiratoires de suspensions de tubules proximaux de chien (colonne du centre), de hamster (colonne de gauche) ou de lapin (colonne de droite). La contribution relative de la pompe à sodium ( $\mathrm{Na}^{+}, \mathrm{K}^{+}$-ATPase sensible à la ouabaïne) et de la pompe à protons $1 H^{+}-A T P a s e$ insensible à la ouabaine mais sensible à la bafilomycine) à la respiration cellulaire phosphorylante (sensible à l'oligomycine) est indiquée dans les boîtes. La partie inférieure des colonnes représente la respiration physiologiquement découplée (insensible à l'oligomycine). La respiration inhibée par le DMSO seul (véhicule de la bafilomycine) est indiquée. Les mesures ont été effectuées en présence des substrats exogènes appropriés pour combler les exigences métaboliques de chaque espèce : lactate : pyruvate $(10: 1 \mathrm{mM})$ et glutamine : glutamate $(10: 1 \mathrm{mM})$ pour le chien et le hamster; lactate :pyruvate (10:1 mM) et glucose (10 mM) pour le lapin. 
$\mathrm{pH}$ influençaient l'activité hydrolytique de l'ATP, le transport de protons par des vésicules de $\mathrm{MBB}$ et la synthèse d'ATP de tubules proximaux de chien [22, 26].

3) L'activité de la pompe pourrait aussi être modifiée par de petits changements de concentration d'ATP (son substrat) ou d'ADP (un inhibiteur naturel de cette activité) dans le microenvironnement de la pompe. Des hypothèses prévoyant l'existence de gradients intracellulaires de concentration de ces deux nucléotides entre les sites d'utilisation membranaires d'ATP et les sites de rephosphorylation mitochondriale assigneraient aux fluctuations cellulaires en ATP, mais surtout en ADP, un rôle régulateur sur l'activité de la pompe à protons (revue par [2]). Certaines pathologies du transport proximal de bicarbonate, telle l'acidose tubulaire proximale liée au syndrome de Fanconi, pourrait être dues à une phosphorylation mitochondriale ou cytoplasmique moins efficace et à un niveau élevé d'ADP dans la proximité des pompes à $\mathrm{H}^{+}$luminales.

4) Une régulation significative pourrait être aussi exercée par la concentration en bicarbonate dans le liquide tubulaire. En effet, dans les états d'alcalose où la charge tubulaire en bicarbonate n'est pas normalisée ou abaissée par une diminution de la filtration glomérulaire, la charge de bicarbonates filtrés peut devenir importante alors que le mécanisme d'échange $\mathrm{Na}^{+} / \mathrm{H}^{+}$est virtuellement inhibé par l'élévation du $\mathrm{pH}$ intracellulaire [32]. Pourtant, la réabsorption des bicarbonates continue ou même s'accroît. On pourrait envisager une influence directe des bicarbonates filtrés sur le canal à proton de la

\section{RÉFÉRENCES}

33. Abdelkhalek MB, Barlet C, Doucet A. Presence of an extramitochondrial anionstimulated ATPase in the rabbit kidney: localization along the nephron and effect of corticosteroids. I Membr Biol 1986;89 225-40.

34. Stone DK, Xie XS. Proton translocating ATPases: Issues in structure and function. Kidney Int 1988 ; 33 : S767-S74. pompe (complexe lipophile), facilitant la sécrétion luminale de protons et donc le processus de réabsorption.

5) Enfin, certains effets inhibiteurs ou stimulateurs exercés sur cette pompe par différents anions organiques suggèrent qu'un site de régulation anionique, situé sur le versant cytoplasmique de la pompe, puisse également contribuer à sa régulation $[6,25,33]$. Les anions physiologiquement impliqués ici ne sont pas encore définis.

6) Il faut mentionner que certaines hormones (hormone parathyroïdienne, angiotensine II, etc.) capables d'activer différents signaux intracellulaires pourraient modifier, soit le trafic vésiculaire, soit l'activité intrinsèque des pompes à protons déjà insérées dans la MBB du tubule proximal.

7) D'autres influences tels le potentiel membranaire [1], la composition lipidique de la MBB [34] et l'activité des échangeurs anioniques basolatéraux (revue par [1]) pourraient enfin participer directement ou indirectement à la régulation de la sécrétion de protons par la pompe à protons luminale.

\section{Conclusion}

On doit donc admettre que l'activité d'une pompe ATP-dépendante présente sur dans MBB est un mécanisme potentiellement important de sécrétion de protons dans le tubule proximal de certaines espèces, en particulier chez le chien utilisé ici comme représentant des carnivores. On peut envisager que l'existence d'une pompe à protons sur la $\mathrm{MBB}$, assurant une réabsorption maximale de bicarbonate et surtout une sécrétion précoce d'acide, soit importante chez les espèces générant des déchets métaboliques acides en quantité importante et variable. Si on peut extrapoler de nos observations faites chez le lapin et le hamster, ce mécanisme serait moins important chez les herbivores. Chez ces espèces, ce mécanisme serait cependant utile au cours des situations d'alcalose métabolique ou respiratoire. En effet l'échangeur $\mathrm{Na}^{+} / \mathrm{H}^{+}$, un mécanisme puissant de sécrétion de protons dans les conditions physiologiques et stimulé par l'acidose métabolique, est virtuellement inhibé en situation d'alcalose. La pompe à protons devient peut-être alors le principal mécanisme de défense du maintien de la concentration extracellulaire de bicarbonate. Une compréhension approfondie des mécanismes de régulation de l'expression génétique de cette pompe, de sa répartition membrano-vésiculaire et de la régulation de son activité intrinsèque modifiera peut-être notre interprétation de la réponse rénale aux alcaloses et aux acidoses. Ces nouvelles notions jetteront sans doute un éclairage nouveau sur la genèse des différents types d'acidoses tubulaires proximales

\section{Summary}

A proton pump in the proximal brush-border membrane

The proximal tubule of the nephron is responsible for considerable bicarbonate reabsorption. This transport is achieved indirectly through net secretion of protons. Three mechanisms are capable of $\mathrm{H}^{+}$secretion in the tubular lumen. Amongst them, a specific $\mathrm{H}^{+}$pump is thought to contribute to this function but the magnitude of its contribution is undefined. We have demonstrated in the dog BBM the presence of a protein capable of transporting $\mathrm{H}^{+}$upon energization by ATP. This $\mathrm{H}^{+}$pump presents the pharmacologic characteristics of a vacuolar $\mathrm{H}^{+}$-ATPase. We have examined the metabolic cost of $\mathrm{H}^{+}$secretion through this mechanism in intact dog tubules in suspension. We demonstrate that this activity is a major ATP-requiring process, requiring nearly $50 \%$ of the local ATP turnover. The activity of this pump is modulated by intracellular $\mathrm{pH}$ and by several other modes of regulation. Theoritical considerations on its putative implication in human diseases such as tubular acidosis type II and Fanconi syndrome are provided.

\section{TIRÉS A PART}

P. Vinay. 